\title{
An Overview of Supervised Machine Learning Techniques in Evolving Mancala Game Player
}

\author{
Randle Oluwarotimi Abayomi ${ }^{1}$, Olugbara O. \\ Oludayo $^{2}$ \\ ${ }^{1}$ Department of Computer Science, \\ Tshwane University of Technology, \\ Pretoria, South Africa \\ ${ }^{2}$ Department of Information Technology, \\ Durban University of Technology, \\ Durban, South Africa
}

\author{
Lall Manosh ${ }^{3}$ \\ Department of Computer Science, \\ Tshwane University of technology, \\ Pretoria, South Africa
}

\begin{abstract}
-supervised machine learning techniques have been employed to evolve Awale game players. This paper studies the various types of supervised learning techniques and views the performance against the Awale shareware. It also recommends a new benchmark for the game.
\end{abstract}

Keywords-supervisedt; machine learning; Awale; grandmaster; game

\section{INTRODUCTION}

A game is an event in which one or more people compete against each other or a computer (simulated player) to get a desired result or learn a game. It could also be the act of testing the strength of certain players against new players. Generally a game is an event that brings fun and entertains individuals. Machine learning as an aspect of Artificial Intelligence (AI) has provided the opportunity for humans to compete against computer evolved players and watch computer compete against computer [1]. These computer games include Chess, Awale, Poker and Bagamonn and are games children and adults play regularly and competitively. The game of Awale has become a widely known game across the world, especially with several researchers evolving players of the games using various techniques. The game is known by certain names across the world such as Ayo, Awele, Omweso, Abapa, Adi and all the various variants belong to the family of mancala games [2].

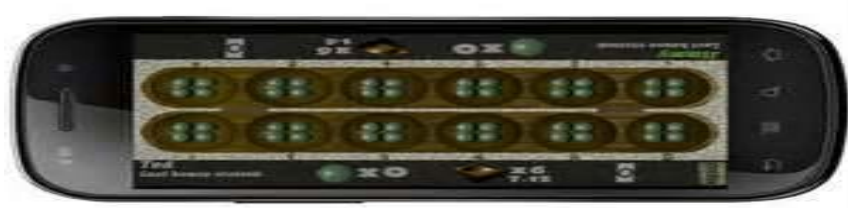

Fig. 1 Example of an Awale game

The description of this combinatorial count-and-capture, two -person-zero-sum board game include the fact that it comprises 12 pits on two rows called as usual, North and South, with 4 seeds in each pit at the beginning of a game [3]. The rules applied include a player selects all seeds from a non-empty pit on his row and sows them counter- clockwise into each pit excluding the starting pit. If the last seed is sown into a pit on the opponent's row, leaving that pit with 2 or 3 seeds, the player captures the seeds in the pit and seeds in preceding pits on the opponent's row that contain 2 or 3 seeds (this is called the 2-3 capture rule). A player cannot capture all the seeds on the opponent's row, so he is obliged to make a move that will give his opponent a move and this is called the golden rule. A controversial rule of Awale, yet to be resolved, is when a player cannot move in such a way that he gives his opponent a legal move, then either the game is cancelled or the player that caused this stalemate loses the game no matter his score. The game comes to a conclusion if one of the 3 events occur:

- $\quad$ when a player has captured more than 24 seeds, or

- when both players have captured 24 seeds leading to a draw or

- $\quad$ when fewer seeds circulate endlessly on the board. Case (3) has the following specialisation: if there are fewer seeds on the board that neither player can ever capture, but both players will always have a legal move, the game ends and each player is awarded the seeds on his row.

There have been various techniques which have been used to evolve Awale game players and all have produced different results when playing competitively against other computer models of the same game or against the Awale shareware [4]. These techniques can be divided into supervised machine learning techniques and unsupervised machine learning techniques. Supervised learning techniques are techniques which have predetermined classification and these classes can be conceived of as a finite set which was previously arrived at by a humans and can also be defined as the process of searching for algorithms that reason from externally supplied instances to produce general hypothesis which then makes predictions about future events [5] while unsupervised learning techniques are not provided with classifications and the basic concept of unsupervised learning is to develop classification labels automatically or can be defined as the attempt to uncover hidden regularities or similarities or detect anomalies in the data [6]. Unsupervised learning is also known as cluster analysis and the machine and aims at 
finding similarities between pieces of data [5,7]. This study focuses on supervised learning techniques and the results which have been obtained from the various implementations in the game of Awale.

\section{SUPERVISED MACHINE LEARNING}

This learning process in supervised machine learning has stages which must be followed for it to be applied to real life scenarios. The first of these stages is data collection. Data collection is the process of acquiring all the relevant elements which would be used for the process. If there is an expert for collecting data then the expert determines the fields or characteristics have relevant data(information) else brute force is applied to gain the necessary data with the assumption that relevant features or characteristics can be obtained[5]. There is need to understand that data obtained by brute force might not be suitable for induction as they may contain missing values or features [ 8] and therefore must be re-processed more than once to acquire the best from it. The second stage is the pre-processing of data and there have been several techniques used to manage and reconstruct missing or lost data [9, 10]. Others uses techniques that handle noise in small data sets but cannot handle very large dataset such as instance selection [11] while some procedures are designed for large datasets [12]. Another technique is Feature subset selection (FSS) which identifies and removes irrelevant and unnecessary features [13]. The next process is defining the training set which is the process of categorising the training sets into their various field and divisions. The process of selecting the best algorithm is a vital and significant step and is determined once initial testing has been proved to be adequate and sufficient then a classifier is available for usage. This unique classifier must be accurate and there are 3 sets to techniques to determine the accuracy [5]. The techniques are

- $\quad$ Split the training set by using two-third for training and the left over for estimating performance

- The training set is separated equally and therefore the average error rate of each subset is calculated and is therefore an estimate of the error rate of the classifier. This is called cross-validation.

- Thirdly is the leave one out validation which is a variation of the second technique.

Supervised learning techniques can furthermore be divided into logical based algorithm such as decision trees [14], Perceptron-based techniques such as single layered perceptrons [15], multilayered perceptrons [16] and Radial basis function network [17],Statistical Learning Algorithms such as Naïve Bayes Classifiers[18], Bayesian networks [19]. Instance Based learning and Support Vector Machines are the other divisions of supervised learning technique.

Supervised Machine Learning Techniques Used To Evolve Awale Player Several supervised machine learning techniques have been used to evolve Awale game players this section will discuss them any analyze the interesting results which were obtained from their experiment.

\section{A. Refinement Assisted Minimax (RAM))}

The basic idea behind the Refinement Assisted Minimax (RAM) algorithm [28] was to find a new strategy similar to the fictitious strategies where their efficiency depended on how efficient was the given strategy. Fictitious play was originally introduced in [20] and it is the most studied process for games [21] and a very good example is the End Game Tchoukailon (EGT) positions [22, 23].

Therefore there was need for a good mechanism that would supports bluffing and formation of Kroo [23] or Odu $[24,25,22]$ in Ayo. EGT did not model the Odu concept but it could readily be used to design strong play heuristics. The study realized that such strategies were practically unrealistic to configure especially when many seeds were on the board but they were useful tools for heuristics design. RAM implemented 3 refinement algorithms.

The first algorithm was called Basic Refinement procedure (BRP) which had a simple myopic rule of play which were:

(a) Given a game state, let the vector move $\mathrm{V}[\mathrm{k}]=\left\{m_{1}, m_{2}, \ldots, m_{k}\right\}$ be a set of $\mathrm{K}$ feasible moves. This is called $m_{k}$ the head and $m_{1}$ the tail. A move is protected if it is not vulnerable to being fortified when the antagonist plays.

(b) If $\mathrm{K}=1$ Then select the only available move and stop.

(c) If tail/head is not protected for South/North player respectively. Then select it otherwise select a move with the highest mobility strength.

The second algorithm was called Priority, where the moves were classified in 2 classes which were $C_{1}$ and $C_{2}$ which were classes of moves that give the player EGT advantage and class of moves that give the opponent EGT advantage respectively using the on-line perceptron learning algorithm [26].

$w_{k+1}=\left\{\begin{array}{l}w_{k}+n y_{i}, \text { if } g(x)<0 \text { and } y_{i} \in C_{1} \\ w_{k}-n y_{i}, \text { if } g(x) \geq 0 \text { and } y_{i} \in C_{2}\end{array}\right.$

Where $y_{i}$ is an example that has been misclassified by the weight $w_{k}, \eta$ is the learning rate and for a given threshold weight $W_{0}, g(x)$ is a linear discriminant function of the input vector $\mathrm{x}$ given by the following equation.

$$
g(x)=w_{0}+\sum_{i=1}^{m} w_{i} x_{i}
$$


The feature vectors in $\boldsymbol{C}_{1}$ were given higher priority than those in $C_{2}$ and a vector in $C_{1}$ whose distance were farthest from the separating hyper-plane was selected [26]. The studied ensured that if all the vectors were found in $\boldsymbol{C}_{2}$, then BRP algorithm was applied. This algorithm was described more compactly by the following pseudo-code.

(1) Let $X_{1}, X_{2}, \ldots ., X_{n}$ be moves recommended by Minimax algorithm.

(2) Classify these moves into $\boldsymbol{C}_{1}$ and $\boldsymbol{C}_{2}$ classes using the perceptron learning.

(3) If $C_{1}$ is not empty Then select the $K$ moves satisfying $\quad X_{k}=\max \left\{g\left(V\left(X_{k}\right)\right)\right\} \quad$ where $V\left(X_{k}\right)$ is the feature vector [37] corresponding to the strategy $X_{k}$ store them in the array move $[\mathrm{m}]$ and store the dimension of move[m] as $\mathrm{m}$. (4) Else select all moves in $\boldsymbol{C}_{2}$, store them in the array move $[\mathrm{m}]$ and store the dimension of move $[\mathrm{m}]$ as $\mathrm{m}$.

(5) Apply BRP to the moves stored in move[m] array.

The third algorithm implemented by RAM is called 'Casing' which combines Case-Based Reasoning (CBR) [28] method with perceptron learning as the basic move classification algorithm.

At the training phase, the method determined the source episodes which are the closest neighbourhood to the target episode. The similarity, $\operatorname{sim}\left(X_{i}\right.$ and $\left.y_{j}\right)$ between two episodes $X_{i}$ and $y_{j}$ was calculated using the following product-moment formula for linear correlation coefficient [29].

$$
\operatorname{sim}\left(\boldsymbol{X}_{i}, \boldsymbol{y}_{j}\right)=\frac{\sum_{k=1}^{m}\left(\boldsymbol{X}_{i k}-\boldsymbol{X}_{a i}\right)\left(\boldsymbol{y}_{j k}-\boldsymbol{y}_{a j}\right)}{\sqrt{\sum_{k=1}^{m}\left(\boldsymbol{X}_{i k}-\boldsymbol{X}_{a i}\right)^{2} \sum_{k=1}^{m}\left(\boldsymbol{y}_{j k}-\boldsymbol{y}_{a j}\right)^{2}}}
$$

To evaluate the performance of each of the refinement algorithm, OPON played a series of games against Awale. They register to play against the Awale program but the initial level was free. At the initiation level using the 3 refinement algorithms OPON captured majority of the seeds after 19, 18 and 27 moves. At the beginner level OPON defeated Awale on the average of 38, 38 and 37 moves. At the Amateur level OPON defeated Awale, although Awale was defeated once at the beginner level and twice at the amateur level with one draw using priority algorithm but was not able to defeat Awale using the casing algorithm. The results of the results at the amateur and grandmaster stage are shown in Table 1.

Table I. The refinement process

\begin{tabular}{|l|l|l|l|}
\hline \multicolumn{4}{|c|}{ BPR } \\
\hline LEVEL & $\begin{array}{l}\text { AVERAGE } \\
\text { MOVES(STD) }\end{array}$ & $\begin{array}{l}\text { SEEDS } \\
\text { CAPTURED } \\
\text { BY EVOLVED } \\
\text { PLAYER(STD) }\end{array}$ & $\begin{array}{l}\text { SEEDS } \\
\text { CAPTURE } \\
\text { D } \\
\text { AWALE(S } \\
\text { TD) }\end{array}$ \\
\hline AMATEUR & $70.50(22.1)$ & $23.83(2.56)$ & $17.00(4.00)$ \\
\hline $\begin{array}{l}\text { GRANDMAS } \\
\text { TER }\end{array}$ & $36.50(10.1)$ & $6.67(3.33)$ & $31.00(4.90)$ \\
\hline \multicolumn{4}{|c|}{ PRIORITY } \\
\hline AMATEUR & $41.83(9.30)$ & $21.83(6.21)$ & $18.17(8.01)$ \\
\hline $\begin{array}{l}\text { GRANDMAS } \\
\text { TER }\end{array}$ & $43.17(3.13)$ & $13.33(7.17)$ & $31.67(4.08)$ \\
\hline \multicolumn{4}{|c|}{ CASING } \\
\hline AMATEUR & $48.33(18.8)$ & $25.17(0.41)$ & $14.17(1.60)$ \\
\hline $\begin{array}{l}\text { GRANDMAS } \\
\text { TER }\end{array}$ & $41.50(2.74)$ & $25.50(0.55)$ & $15.00(1.00)$ \\
\hline
\end{tabular}

\section{B. Cased based reasonings}

Case based reasoning is the process of using an old technique or experience to solve a new problem. This technique uses a reasoned which assists by remembering the previous problem and the solution which was used to solve the problem [29]. At the testing phase are a new episode is discovered its similarities of the source episodes are calculated, where the similarity between ith target episode $\boldsymbol{X}_{i}=\left(\boldsymbol{X}_{i 1}, \boldsymbol{X}_{i 2}, \boldsymbol{X}_{i 3}, \ldots, \boldsymbol{X}_{i m}\right)$ and the source episode $y_{j}=\left(y_{j 1}, y_{j 2}, y_{j 3}, \ldots . ., y_{j m}\right)$ of the th class is computed. Note - That the target episode with game value $\leq \alpha$ and similarity measure $\geq \beta$ was selected. The similarity was denoted by $\operatorname{Sim}\left(X_{i}, Y_{j}\right)$ between $X_{i}$ and $y_{j}$ was calculated using the product-moment formula for the linear correlation coefficient. The similarity was defined as

$$
\operatorname{sim}\left(\boldsymbol{X}_{i}, \boldsymbol{y}_{j}\right)=\frac{\sum_{k=1}^{m}\left(\boldsymbol{X}_{i k}-\boldsymbol{X}_{a i}\right)\left(\boldsymbol{y}_{j k}-\boldsymbol{y}_{a j}\right)}{\sqrt{\sum_{k=1}^{m}\left(\boldsymbol{X}_{i k}-\boldsymbol{X}_{a i}\right)^{2} \sum_{k=1}^{m}\left(\boldsymbol{y}_{j k}-\boldsymbol{y}_{a j}\right)^{2}}}
$$

Where $\boldsymbol{X}_{a i}$ and $\boldsymbol{y}_{a j}$ are the average values of $\boldsymbol{X}_{i}$ and $\boldsymbol{y}_{j}$, respectively, and $\mathrm{m}$ is the number of pits on the Ayo board. Furthermore a tournament was conducted between Minimax, Minimax-CBR and Awale and the results are shown in Table 2 [33]. 
Table II. $\quad$ Case Based Reasoning

\begin{tabular}{|l|l|l|l|}
\hline MINIMAX(STD) & AWALE(STD) & MOVES(STD) & $\begin{array}{l}\text { OVERRID } \\
\text { ES }\end{array}$ \\
\hline $16.00(5.27)$ & $26.50(0.53)$ & $68.00(45.33)$ & $\begin{array}{l}\text { NOT } \\
\text { APPLICA } \\
\text { BLE }\end{array}$ \\
\hline \multicolumn{3}{|c|}{} \\
\hline MINIMAX & $\begin{array}{l}\text { MINIMAX- } \\
\text { CBR }\end{array}$ & MOVES & $\begin{array}{l}\text { OVERRI } \\
\text { DES }\end{array}$ \\
\hline $7.00(3.16)$ & $28.00(3.16)$ & $38.50(11.92)$ & $\begin{array}{l}10.10(2.2 \\
3)\end{array}$ \\
\hline \multicolumn{3}{|l}{} \\
\hline MINIMAX-CBR & AWALE & MOVES & $\begin{array}{l}\text { OVERRI } \\
\text { DES }\end{array}$ \\
\hline $25.50(0.53)$ & $15.00(1.05)$ & $42.70(2.31)$ & $\begin{array}{l}24.00(2.1 \\
1)\end{array}$ \\
\hline
\end{tabular}

\section{Linear discriminant Algorithm(LDA)}

It analyses a computer player as a hyperplane in which the input space minimizes the within-class variance and maximizes the between class distance [34]. It computes efficiently in linear cases especially with large data sets. The LDA function is defined in equation 5. [38] evolved an Awale player using the technique and applying the mathematical equation to derive the players.

$$
\mathrm{g}(\mathrm{x})=w 0+\sum_{i=1}^{n} w_{i}+x_{i}
$$

Where,

$w$ is the weight vector

$w_{0}$ is the bias or threshold weight

$x_{i}$ is the feature vector.

The augmented weight vector w1was estimated based on the available training data. Also the study used a linear classifier which categorises Awale strategies into 2 disjoint classes. The 2 classes were classes of strategies that give a player Tchoukaillion positions [35] and secondly the class of strategies that give opponent tchoukaillon advantage.

\section{TABLE III. LINEAR DISCRIMINANT ALGORITHM}

\begin{tabular}{|l|l|l|l|}
\hline Game & $\begin{array}{l}\text { Average } \\
\text { moves }\end{array}$ & $\begin{array}{l}\text { Average } \\
\text { seeds } \\
\text { captured by } \\
\text { evolved } \\
\text { player }\end{array}$ & $\begin{array}{l}\text { Average } \\
\text { Seeds } \\
\text { captured } \\
\text { by Awale }\end{array}$ \\
\hline INITIATION & 23 & 26 & 8.7 \\
\hline BEGINNER & 24 & 26 & 7.6 \\
\hline AMATEUR & 20 & 26 & 25 \\
\hline GRANDMASTER & 29 & 13.4 & 25 \\
\hline
\end{tabular}

The results of the player evolved using LDA showed that the player performed well in the first 2 stages against the Awale shareware. It also won 3 of the 5 games played at the Amateur level and lost all games against the Grandmaster.

\section{Co-evolutions}

Awale game player is evolved using the approach where computer compete against each other , thereby leaving the strongest set of players to be mutated with the aid of evolutionary strategies. The study wanted to investigate if the evaluation functions would improve the performance of their evolved player. The study used 6 evaluation functions [36] and used the function in equation below as the basis. The results of the experiment showed that the evaluation function did not improve the performance. The function used to represent the evolved player were

$f=w_{1} a_{2}+w_{2} a_{3}+w_{3} B_{2}+w_{4} B_{3}+w_{5} a_{5}+w_{6} B_{s}(6)$ where:

$W_{1} \cdots W_{6}$ The weight for each term of $f$

$\boldsymbol{a}_{2}$ The number of the opponent's pits vulnerable to having 2 stones captured on the next move

$\boldsymbol{a}_{3} \quad$ The number of the opponents pits vulnerable to having 3 stones captured on the next move

$B_{2}$ The number of the evolving players pits vulnerable to having 2 stones captured on the next move

$\boldsymbol{B}_{3}$ The number of the evolving players pits vulnerable to having 3 stones captured on the next move

$\mathrm{a}_{\mathrm{s}} \quad$ The current score of the opponent

$b_{s} \quad$ The current score of the evolving player

TABLE IV. C0-EVOLUTION

\begin{tabular}{|c|c|c|c|}
\hline Level & $\begin{array}{c}\text { AVERAGE } \\
\text { MOVES }\end{array}$ & $\begin{array}{c}\text { SEEDS WON } \\
\text { BY } \\
\text { EVOLVED }\end{array}$ & $\begin{array}{c}\text { SEEDS WON } \\
\text { BY AWALE }\end{array}$ \\
\hline INITIATION & 47.40 & 29.80 & 2.80 \\
\hline BEGINNER & 55.80 & 26.20 & 7.80 \\
\hline AMATEUR & 108.20 & 24.20 & 6.80 \\
\hline GRANDMASTER & 80 & 4.40 & 6.80 \\
\hline
\end{tabular}

\section{E Genetic Algorithm}

The unique technique was used by [37] to evolve the Awale game player with the aim of proving that increasing the number of features in the evaluation function can lead to a reduction in the mini-max search depth. The study used the equation below as their evaluation function .the study developed 6 new features to improve their evaluation function and added d 6 used in co-evolution [36]. The genetic algorithm implemented had 6 operations which were Problem encoding, fitness evaluation, selection and elitism, crossover, mutation and termination criteria. The result obtained from the evolved player did not improve its performance.

Their study reduced the response time, the CPU usage and the amount of memory required during the evaluation. Daoud et al's evaluation function was 


$$
f=\sum_{i=1}^{12} w_{i} \times a_{i}
$$

The twelve features used in his study are were

W1..w12 The weights of each term of $\mathrm{f}$. They range between $[0,1]$.

a1 The number of pits that the opponent can use to capture 2 seeds. Range: 0-6.

a2 The number of pits that the opponent can use to capture 3 seeds. Range: 0-6.

a3 The number of pits that Ayo can use to capture 2 seeds, range: $0-6$.

a4 The number of pits that Ayo can use to capture 3 seeds. Range: 0-6 .

a5 The number of pits on the opponent's side with enough seeds to reach to Ayo's side. Range: 0-6.

a6 The number of pits on Ayo's side with enough seeds to reach the opponent's side. Range:0-6

a7 The number of pits with more than 12 seeds on the opponent's side .Range: 0-6.

a8 The number of pits with more than 12 seeds on Ayo's side. Range: 0-6

a9

The current score of the opponent. Range:0-48

a10 The current score of Ayo. Range: 0-48.

a11 The number of empty pits on the opponents side. Range:0-6.

a12

The number of empty pits on Ayo's side. Range: 0-6

This study used genetic algorithm to evolve their weights which was used for their evaluation function. Table 5 shows the performance of the evolved player in which it performed well at the 3 initial stages but lost the final (grandmaster stage).

TABLE V. GENETIC ALGORITHM

\begin{tabular}{|l|l|l|l|}
\hline LEVEL & $\begin{array}{l}\text { AVERAGE } \\
\text { MOVES }\end{array}$ & $\begin{array}{l}\text { AVERAGE } \\
\text { SEEDS } \\
\text { CAPURED } \\
\text { BY } \\
\text { EVOLVED } \\
\text { PLAYER }\end{array}$ & $\begin{array}{l}\text { AVG } \\
\text { SEEDS } \\
\text { CAPURED } \\
\text { BY } \\
\text { AWALE }\end{array}$ \\
\hline INITIATION & 53.60 & 26.5 & 8.3 \\
\hline BEGINNER & 121.6 & 26.3 & 11.7 \\
\hline AMATEUR & 140 & 24.8 & 15.5 \\
\hline GRANDMASTER & 137 & 5.3 & 28.8 \\
\hline
\end{tabular}

\section{CONCLUSION}

This study has investigated the performance of various supervised machine learning techniques used to evolve Awale game player. It has come to the conclusion that Casebased reasoning (CBR) and the refinement procedure
(Casing) should be used as benchmarks for the game since we know the technique which they were developed upon rather than the Awale Shareware (whose technique is being kept a secret).

\section{ACKNOWLEDGMENT}

I would like to thank my mentor Prof O.O Olugbara for his expert contributions to this paper.

\section{REFERENCES}

[1]Hamilton, S and Graber, (1997) Deep Blue hardware-software synergy. IEEE computer 30, 10,pp29-35

[2]Agbinya , J.I (2004) Computer Board games of Africa(Algorithm ,Strategies and Rules). Available at http://services .eng.uts.edu.au/agbinya/computer/20games/African Board games. Pdf.Accessed 23/10/2010.

[3]Adewoye, T.O (1990) On Certain Combinatorial Number Theoretic Aspects of the African Game of 'Ayo'. AMSE Review, 14(2), 41-63..

[4]Myraid software.http://www.myraid-online.com/awale.htm

[5]Kotsiantis, 2007 Supervised machine Learning: A Review of Classification Technique. Informatica 31, 249-268

[6]Meir and Ratsch, 2003., An introduction to boosting and leveraging. Paper presented at the in Proceeding of the Twenty -First International Conference on Machine Learning.

[7]Jain , A K., Murty, M.N and Flynn, P. (1999). Data Clustering: A review, ACM Computing Surveys, 31(3): 264-323.

[8]Zhang .S., Zhang, C and Yang,Q( 2002). Data Preparation for Data Mining. Applied Artificial Intelligence, Volume 17, pp 375-381

[9]Batista and Monard, 2003. An Analysis of Four missing Data Treatment Methods for Supervised Learning, Applied Artificial Intelligence, Vol 17, pp 519-533.

[10]Hodge and Austin, (2004). A Survey of Outlier Detection Methodologies, Artificial Intelligence Review, Volume 22, Issue 2, pp. 85126

[11]Liu and Motoda, (2001). Instance Selection and Constructive Data Minimg, Kluwer , Boston,

[12]Reinartz, T (2002). A Unifying View on Instance Selection, Data Mining and Knowledge Discovery, 6, 191-210, Kluwer Academic Publishers

[13]Yu , L and Liu, H( 2004). Efficient Feature Selection via Analysis of Relevance and Redundancy, JMLR, 5(Oct): 1205-1224.

[14]Murthy, 1998), Automatic Construction of Decision Trees from Data:A Multi-Disciplinary Survey, Data Mining and Knowledge Discovery 2:345389

[15]Kivinen, J (2002). Online Learning of Linear Classifiers, Advances Lecture on Machine Learning: Machine Learning Summer school 2002. Australia, Febuary 11-22, ISSN: 0302-9743, pp. 235-257.

[16]Parekh, R ., and Yang, J and Honavar, V( 2000). Constructive Neural network Learning Algorithms for pattern Classification. IEEE Transaction on Neural networks. 11(2), pp. 436-451.

[17]Robert, J and Howlett L C J(2001). Radial Basis Function networks 2: New Advances in Design

[18]Yang, Y and Webb,G( 2003). On why Discretization Works for NaïveBayes Classifiers, Lecture Notes in Computer Science, Volume 2903, pages $440-452$

[19]Madden (2003.The performance of Bayesian network Classifier Constructed using different techniques . Proceedings of European Conference on Machine Learning, Workshop on Probabilistic Graphical Models for Classification, pp 59-70.

[20]Brown , G.W., Iterative solutions of games by ficticious play, Activity analysis of production and allocation,Koopmans(ed) Wiley, new York, pp. 374-376,1951 
[21]Berger, U., two More Classes of games with the Ficticious Play Property. Game theory and Information 0408003, Economics working paper Archive EconWPA,2004

[22]Broline, D.M. and Loeb, D.E. (1995). The Combinatorics of Mancalatype games: “Ayo", Tchoukaillon and UMAP J., 1(41),1-15.

[23]Donkers, H.H.L.M., Uiterwijk, J.W.H.M. and Voogt, A.J. De (2002).Mancala Games Topics in Artificial Intelligence and Mathematics.Step by Step Proceedings of the $4^{\text {th }}$ Colloquium Board Games in Academia (eds. J. Retschitzki and R. Haddad-Zubel), Editions Universitaires, Fribourg, Switserland. 33-146

[24]Odeleye, A.O., Ayo: A popular Yoruba game, Oxford University Press, Ibadan, Nigeria, 1977

[25]Ayeni, J.O.A and Longe, H.O.D., Game people Play: Ayo. International Journal of Game Theory, Vol.14 issue 4,pp. 207-218, 1985

[26]Jantzen, J.Introduction to Perceptron networks. Tech. report no 98H873, Department of Automatic, technical University of Denmark,1998

[27]Daoud, M., Kharma, N., Haidar, A. and Popoola, J. (2004). Ayo the Awari Player, or How Better Representation Trumps Deeper Search, Proceedings of the 2004 IEEE Congress on Evolutionary Computation, 1001-1006.

[28]Olugbara, O.O., Adewoye, T.O and Akinyemi, I.O (2006). An Investigation of Minimax Search Technique for Evolving Ayo/Awale Player. Proceedings of IEEE-ICICT $4^{\text {th }}$ International Conference on Information and Communication Technology, Cairo, Egypt

[29] koloner, J.L., Case -Base Reasoning. Morgan Kaufmann, 1993.
[30]Kolodner ,J.L., and leake, D., Tutorial Introduction to case-based Reasoning. In: Leake, D,(ed) Case Case-based Reasoning: Experiences, Lessons and future Directions. Pp.31-65.AAAI press /The MIT press, 1996 [31]Spiegel, M.R., Shaum's outline series, theory and problems of statistics. McGraw-Hill Company , 1972

[32] Kolodner, 1992., Case-based Reasoning. Morgan Kaufmann, San meteo.

[33]Olugbara, O.O., Adigun, M.O Ojo, S.O and Adewoye, T, O (2007) Ann Efficient Heuristic for Evolving an Agent in the Strategy game of Ayo. International Computer Games Association Journal, 30, 92-96.

[34]Fisher, 1936., The use of multiple measurements in taxonomic problems. Annals of Eugenics, 7(2):179-188.

[35]Broline, D.M. and Loeb, D.E. (1995). The Combinatorics of Mancalatype games: “Ayo", Tchoukaillon and UMAP J., 1(41),1-15.

[36]Davis, J.E. and Kendall, G. (2002). An Investigation, using Coevolution, to Evolve an AwariPlayer.Proceedings of Congress on Evolutionary Computation (CEC), 1408-1413.

[37]Daoud, M., Kharma, N., Haidar, A. and Popoola, J. (2004). Ayo the Awari Player, or How Better Representation Trumps Deeper Search, Proceedings of the 2004 IEEE Congress on Evolutionary Computation, 1001-1006.

[38 ]Olugbara ,O.O., Gbadeyan, J.A., Adewoye, T.O and Mbatu, K.(2006). Formal Characteristics of Ayo Game Using Linear Discriminant Function and Equivalence Relations. NTMCS Conference Proceedings 